\title{
Differential Diagnosis and Treatment of Isolated Pathologies of the Sphenoid Sinus: Retrospective Study of 46 Cases
}

\author{
Thomas Ribeiro Marcolini $^{1}$ Maryane Cristine Safraider ${ }^{1}$ Jan Alessandro Socher ${ }^{2}$ \\ Guilherme Olinto Lucena ${ }^{3}$ \\ ${ }^{1}$ Department of Medicine, Universidade Regional de Blumenau, \\ Blumenau, SC, Brazil \\ ${ }^{2}$ Department of Otorhinolaryngology, Universidade Regional de \\ Blumenau, Blumenau, SC, Brazil \\ ${ }^{3}$ Department of Medicine, Faculdade Evangélica do Paraná, Curitiba, \\ $\mathrm{PR}$, Brazil \\ Int Arch Otorhinolaryngol 2015;19:124-129.

\begin{abstract}
Address for correspondence Thomas Ribeiro Marcolini, MD, Department of Medicine, Universidade Regional de Blumenau, Rua Colombo 397 apto 05, Cornélio, Procópio PR, Brazil (e-mail: thomasm0603@hotmail.com).
\end{abstract}

\begin{abstract}
Introduction Isolated disease of the sphenoid is rare and has often been overlooked due to its remote location and difficult access.

Objective A retrospective study of the main causes of isolated sphenoid sinus diseases with discussion of the most appropriate methods of diagnosis and treatment.

Methods A total of 46 cases of isolated sphenoid disease treated between January 2008 and December 2013 were evaluated by objective ear, nose, and throat examination and video endoscopy, computed tomography of the paranasal sinuses, and, in some cases, magnetic resonance imaging. In each case, we decided between drug and/or endoscopic treatment.

Keywords

- sphenoid sinus

- paranasal sinuses

- sphenoid sinusitis

- cerebrospinal fluid leak

- paranasal sinus neoplasms

Results We identified 12 cases of isolated sphenoiditis (26.1\%), 3 cases of fungal sphenoiditis (6.5\%), 3 cases of sphenochoanal polyps (6.5\%), 22 cases of mucocele (47.8\%), 2 cases of cerebrospinal fluid leak (4.3\%), and 1 case each of meningoencephalocele (2.1\%), inverted papilloma (2.1\%), fibrous dysplasia (2.1\%), and squamous cell carcinoma (2.1\%).

Conclusion A prevalence of inflammatory and infectious diseases was found, and endoscopic surgery for the sphenoid sinus approach is effective in treating various diseases of the isolated sphenoid, whether complicated or not.
\end{abstract}

\section{Introduction}

Isolated disease of the sphenoid represents 1 to $2 \%$ of all sinuses infections. The sphenoid sinus has often been neglected because of its isolated location and difficult access. ${ }^{1}$ Furthermore, the rarity of sphenoidal involvement can be explained by the nonspecific symptoms, the inaccessibility to the sinus through the otorhinolaryngological physical examination, and the low number of diagnoses prior to the advent of more sophisticated technology, such as computed tomography (CT) and magnetic resonance imaging (MRI). The isolated involvement of the sphenoid sinus in most cases has an inflammatory origin, and a neoplastic origin is rare. ${ }^{2-4}$

Sphenoid sinus diseases are difficult to diagnose and treat, because initial symptoms are vague and difficult to recognize. $^{5}$ The clinical presentation involves headache (mainly retro-orbital), which may be associated with purulent rhinorrhea, retropharyngeal drip, nasal obstruction, abnormal received

October 6, 2014

accepted

November 1, 2014

published online

January 28, 2015
DOI http://dx.doi.org/

10.1055/s-0034-1397337. ISSN 1809-9777.
Copyright $(2015$ by Thieme Publicações License terms Ltda, Rio de Janeiro, Brazil

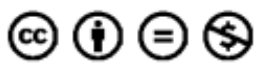


vision, nerve deficits, and consequential damage of other inflammatory or neoplastic processes installed. Delayed diagnosis and treatment can result in serious complications. This is due to the anatomical relations with the brain, meninges, optic nerve, internal carotid artery, cavernous sinus, and cranial nerve (oculomotor, trochlear, ophthalmic, and maxillary branches of the trigeminal nerve and abducens). ${ }^{6}$

Before the introduction of nasal endoscopy and better imaging technologies such as CT and MRI, the deep position of the sphenoid sinus prevented a proper early diagnosis of pathologies there. Moreover, its location in the skull base does not allow a good view through conventional radiographs. Routine use of endoscopic surgery and introduction of sophisticated imaging techniques has allowed more frequent diagnoses of these rare pathologies. ${ }^{7,8}$ The aim of this retrospective study was to survey the leading causes of isolated sphenoid pathologies, with discussion of the most appropriate methods of diagnosis and treatment.

\section{Methods}

We analyzed 46 charts and examination records from January 2008 to December 2013 in patients with isolated sphenoid pathology. At the time of the diagnosis, there was no involvement of other sinuses. Only tumors involving the sphenoid sinus were included. Patients were evaluated by objective ear, nose, and throat examination and by nasal-sinus video endoscopy, 3.2-mm diameter flexible and/or rigid with 30-degree optics. The diagnosis was confirmed by CT of the paranasal sinuses and nasal cavities, in axial and coronal sections, with and without contrast, using a 2,500- to 3,500-rads bone window. In some cases, MRI was used: when there was possible bone erosion of the sinus wall, presence of cerebrospinal fluid leak and visual changes. Isolated sphenoiditis cases without complication were treated with antibiotics (levofloxacin $500 \mathrm{mg} / \mathrm{d}$ ) for 21 days associated with corticosteroid (prednisone $40 \mathrm{mg}$ daily) for 7 days. The patients with worsening or maintenance of symptoms during treatment or within 6 weeks after it received endoscopic surgical treatment. Benign or malignant pathologies (tumor, dysplasia, cerebrospinal fluid leak, mucocele, fungal sphenoiditis) were treated surgically by endoscopic transnasal or transethmoidal sphenoid sinusotomy. Patients were postoperatively followed for at least 6 months for inflammatory diseases and up to 4 years for other benign and malignant pathologies.

\section{Results}

We identified 12 cases of isolated sphenoiditis (26.1\%), in 8 female (66.6\%) and 4 male subjects (33.3\%) from 13 to 73 years of age; 22 of mucocele (47.8\%), in 13 women and 9 men from 18 to 62 years old; 3 of fungal sphenoiditis (6.5\%), in 2 women and 1 man from 33 to 68 years old; 3 of sphenochoanal polyp (6.5\%), in 2 women and 1 man from 30 to 52 years old; 2 of cerebrospinal fluid leak (4.3\%), in 1 female and 1 male patient, 17 and 49 years old; 1 of meningoencephalocele (2.1\%), in a 14-year-old girl; 1 of inverted papilloma (2.1\%), in a 45 -yearold man; 1 of osteofibrous dysplasia (2.1\%), in a 41-year-old woman; and 1 of squamous cell carcinoma (2.1\%), in a 48-year-old man.

The most common symptoms were headache/retro-orbital or frontal facial pain in 33 cases $(71.7 \%)$, nasal obstruction in 15 patients (32.6\%), cerebrospinal fluid rhinorrhea in 7 cases (15.2\%), and mucopurulent rhinorrhea in 6 patients (13.0\%). Also, 2 patients presented with epiphora (4.3\%), 5 with fever (10.9\%), 2 with epistaxis (4.3\%), 1 with diplopia (2.1\%); another patient's level of consciousness was altered and he required hospitalization (2.1\%).

MRI was needed in only 6 cases (13.0\%). Drug treatment was required in 7 cases, sphenoidal sinusotomy in 30, and transethmoidal sphenoidal sinusectomy in 8 . Only 2 cases experienced complications, in which the patients suffered significant bleeding during the transethmoidal sphenoidal sinusectomy, controlled with sphenopalatine artery ligation.

\section{Discussion}

All patients with isolated sphenoiditis reported retro-orbital and/or frontal headache, and 7 had postnasal drip, nasal obstruction, epiphora, and/or fever. There was 1 case of consciousness level decrease. Video endoscopy revealed 7 patients $(58.3 \%)$ with signs of inflammation, characterized by mucosal edema and mucopurulent discharge arising from

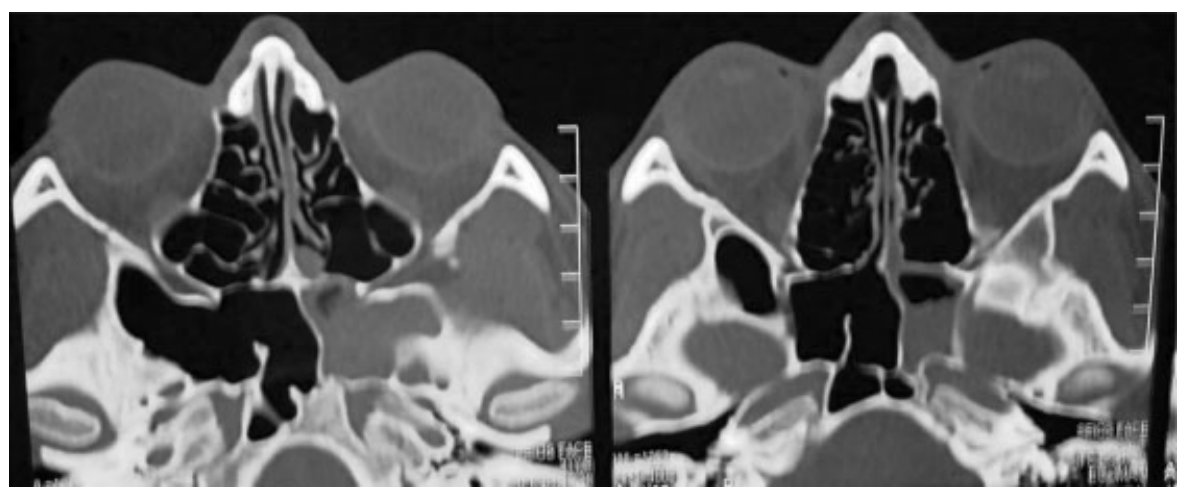

Fig. 1 Axial computed tomography shows sphenoid sinus opacification and greater wing of sphenoid compatible with isolated left sphenoiditis. 


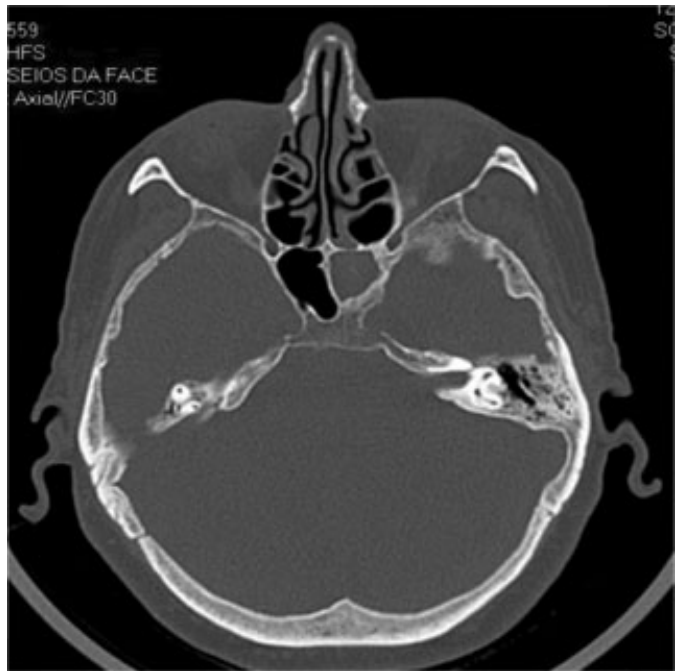

Fig. 2 Axial computed tomography demonstrates homogeneous sphenoid sinus opacification with direct osteitis signs.

the sphenoethmoidal recess. CT revealed an opacity of the sphenoid sinus without any abnormalities in other paranasal sinuses. In 2 cases, CT showed signs of osteitis (-Figs. 1 and $\mathbf{2}$ ), and in 1 case, a sidewall erosion progressed to an extradural empyema (-Fig. 3). Drug therapy with antibiotics and corticosteroids was used in 7 patients, 4 cases were treated by endoscopic transnasal sphenoid sinusotomy, and 1 case was treated through endoscopic transethmoidal sphenoid sinusectomy. Patients who underwent surgery were evaluated with video endoscopy weekly, at 1 month postoperatively, and then monthly thereafter. CT of the paranasal sinuses performed 90 and 180 days after surgery confirmed the absence of sphenoiditis evidence.

In the cases of sphenoid mucocele, the most important symptom was retro-orbital pain. A video endoscopy showed mucosal edema at the sphenoethmoidal recess in only 9

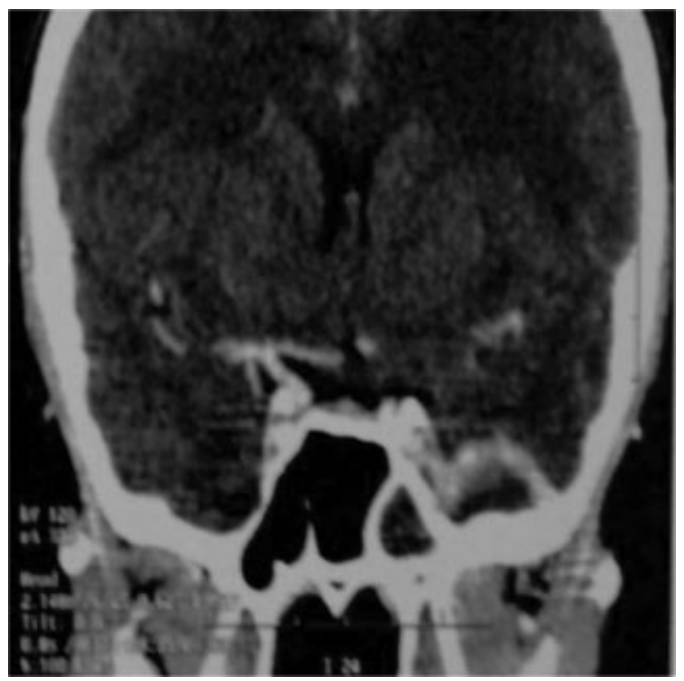

Fig. 3 Preoperative coronal computed tomography shows left sinus opacification evolving to extradural empyema.

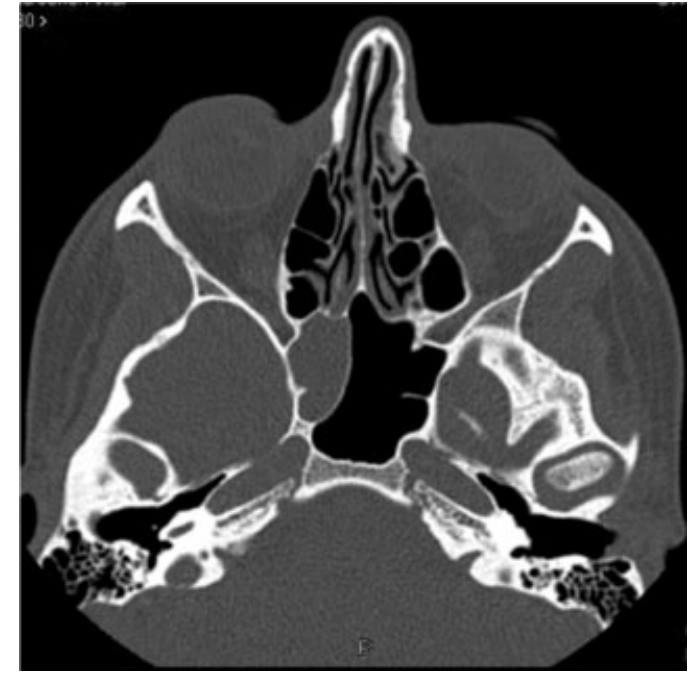

Fig. 4 Preoperative axial computed tomography shows homogeneous opacification on the right sphenoid sinus with no signs of adjacent bone erosion.

patients, and $\mathrm{CT}$ revealed a homogeneous sphenoidal mass without adjacent bone erosion ( - Fig. 4). All patients underwent endoscopic transnasal sphenoid sinusectomy decompression. There were no signs of recurrence at a minimum of 1 -year postoperative follow-up.

Of the 3 patients with fungal sinusitis, 2 presented retroorbital pain and unilateral purulent rhinorrhea, and the other complained only about headache. The endoscopic examination showed edema and purulent discharge at the sphenoethmoidal recess, with postnasal drip. CT in all cases showed heterogeneous images occupying the sphenoid sinus with signs of calcification and osteitis (-Figs. 5 and $\mathbf{6}$ ). Transnasal endoscopic sphenoid sinusotomy treatment was used. The histologic results confirmed Aspergillus presence in all cases. There were no signs of recurrence in all cases with a minimum of 1-year postoperative follow-up.

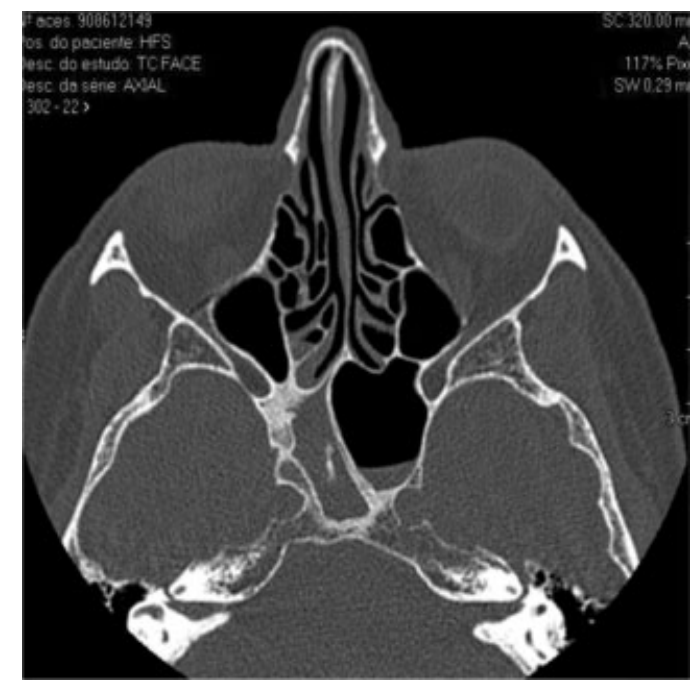

Fig. 5 Preoperative coronal computed tomography shows heterogeneity occupying the right sphenoid sinus with signs of calcification. 


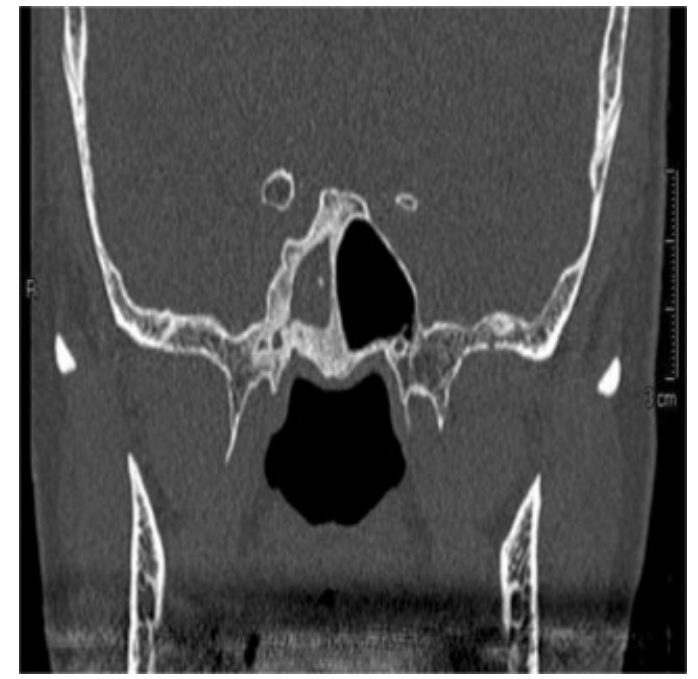

Fig. 6 Preoperative coronal computed tomography shows heterogeneity with calcification signs occupying the right sphenoid sinus with osteitis signs.

The 3 patients with sphenochoanal polyp presented symptoms of unilateral nasal obstruction, and 2 suffered from frontal or retro-orbital headache. Video endoscopy was diagnostic in all 3 cases, revealing polypoid tumor in sphenoethmoidal recess. CT showed an expansive homogeneous image with evidently precise limits in the nasal cavity, with no evidence of bone erosion (-Fig. 6). Patients who underwent endoscopic transethmoidal sphenoidal sinusectomy showed no signs of recurrence for at least 1 year postoperatively.

Of the 2 patients with cerebrospinal fluid leak, 1 reported a previous history of hospitalization because of meningitis. Both patients reported unilateral rhinorrhea and denied previous nasal or sinus surgery and/or cranioencephalic trauma. In all patients, the presence of cerebrospinal fluid

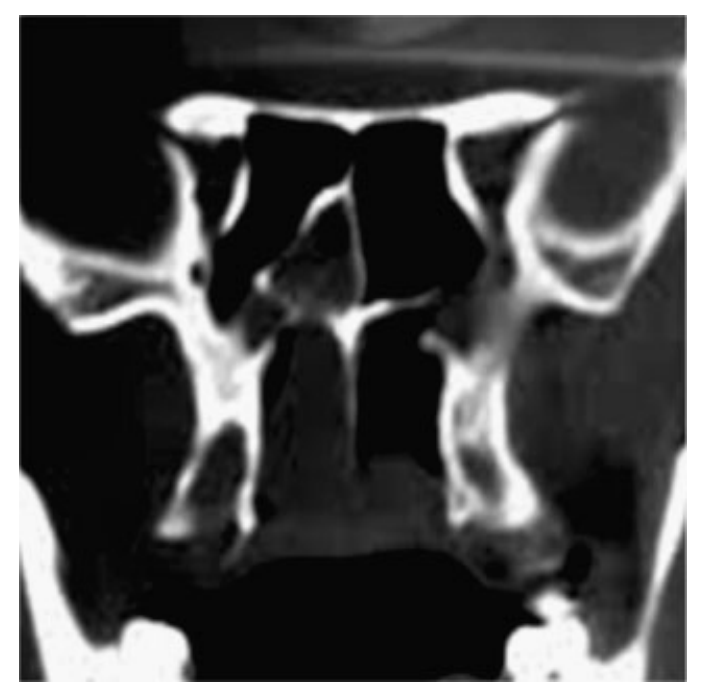

Fig. 7 Preoperative coronal computed tomography demonstrates partial sphenoid sinus opacification and right sphenoid mass occupying precise limits compatible with right sphenochoanal nasal cavity polyp. leak was confirmed by studies of $\beta 2$-transferrin. Endoscopic exams did not show significant changes, and CT demonstrated complete or partial dehiscence with opacification of the sphenoidal sinus wall. MRI showed hyperintense signal on T2 compatible to liquid occupying the sphenoid sinus. All patients underwent endoscopic transnasal sphenoidal sinusotomy to reverse the cerebrospinal fluid leak with free graft of inferior concha mucoperiosteum fixed with fibrin glue. There were no signs of recurrence within postoperative follow-up of 3 years and 2 months.

The main symptoms were nasal in the 14-year-old girl with meningoencephalocele, with obstruction and abundant unilateral rhinorrhea. Video endoscopy revealed the presence of cerebrospinal fluid rhinorrhea from the left sphenoethmoidal recess after the Valsalva maneuver. CT showed a dehiscence of the bone wall with partial opacification of the left sphenoid sinus and meningoencephalic herniation (-Fig. 7). The patient underwent endoscopic transethmoidal sphenoid sinusectomy for correcting the mass protrusion, and the leak was closed with free graft of inferior concha mucoperiosteum fixed with fibrin glue.

The 45-year-old man with inverted papilloma presented with the main complaints of frontal and retro-orbital headache associated with nasal obstruction and right-sided epistaxis. A polypoid tumor in the right sphenoethmoidal recess was identified by endoscopy, and CT revealed a heterogeneous opacification of the sphenoid sinus with contrast uptake, signs of remodeling process of the sphenoid sinus walls, and bone erosion, with no compromise of adjacent structures (-Fig. 8). The patient underwent a bilateral resection of the tumor by endoscopic transethmoidal sphenoid sinusectomy, and the histologic studies confirmed the diagnosis of inverted papilloma. No recurrence was noted at postoperative follow-up of 2 years and 6 months.

The main complaint of the patient with fibrous dysplasia was an intense frontal and retro-orbital left-sided headache.

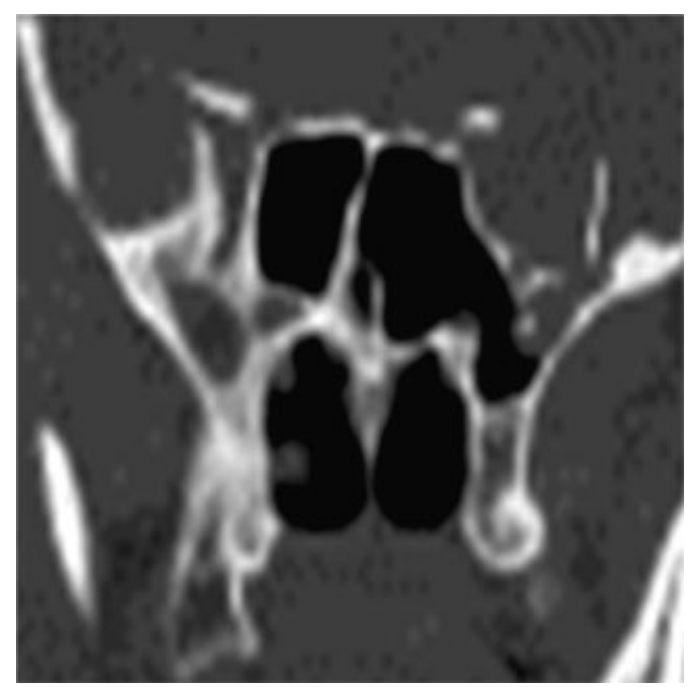

Fig. 8 Preoperative coronal computed tomography section with details showing the sphenoid's lateral wall opacification and dehiscence with partial opacification of the lateral sphenoid's recess. 


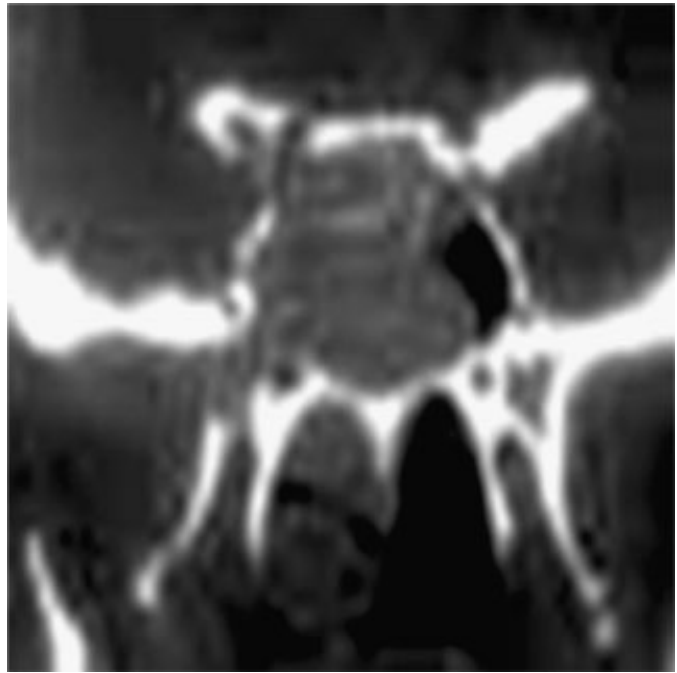

Fig. 9 Preoperative coronal section computed tomography highlighting the right sphenoid heterogeneous opacification with signs of remodeling in the wall of the sphenoid sinus and bone erosion.

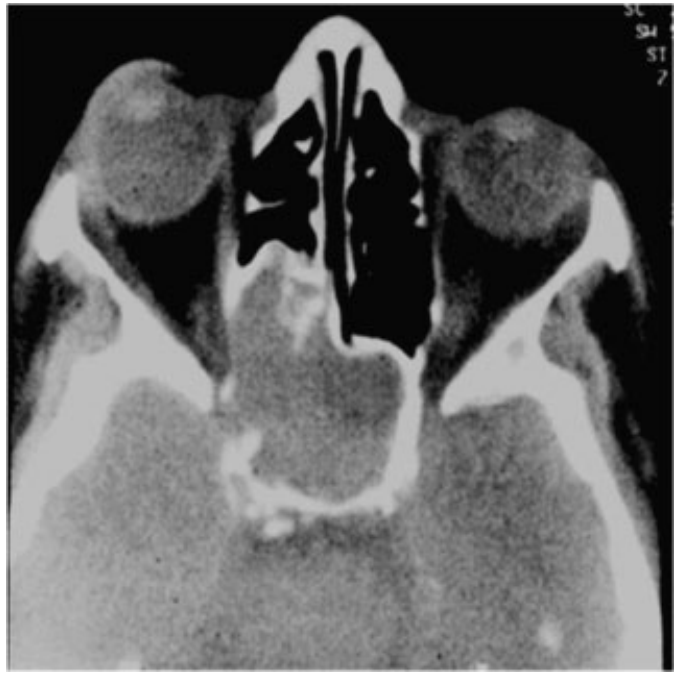

Fig. 11 Preoperative axial computed tomography demonstrates a heterogeneous mass occupying the sphenoid sinuses, erosion of the sinus bony wall, and compression of the left optic nerve.
The endoscopic examination revealed no significant changes, and CT showed areas of thickening and sclerosis occupying

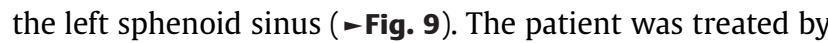
endoscopic transethmoidal sphenoid sinusectomy and showed improvements of the disease symptoms during the postoperative follow-up of 2 years.

In the case of squamous cell carcinoma, the symptoms included retro-orbital headache, nasal obstruction, left-sided diplopia, and eventual epistaxis. The endoscopic examination revealed sphenoethmoidal recess edema, and CT showed an expansive process including erosion of the bone wall of the left sphenoid sinus (-Fig. 10). MRI identified a mass with gadolinium contrast enhancement and confirmed optic nerve compression without infiltration of other structures (-Fig. 11). The patient underwent bilateral transethmoidal sphenoid sinusectomy to resect the tumor with a safe margin

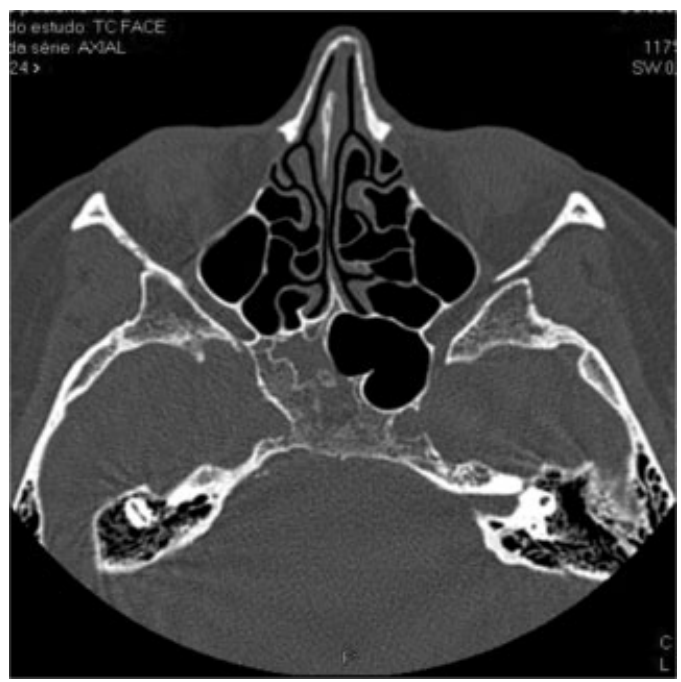

Fig. 10 Preoperative axial computed tomography demonstrating areas of thickening and sclerosis in the left sphenoid sinus.

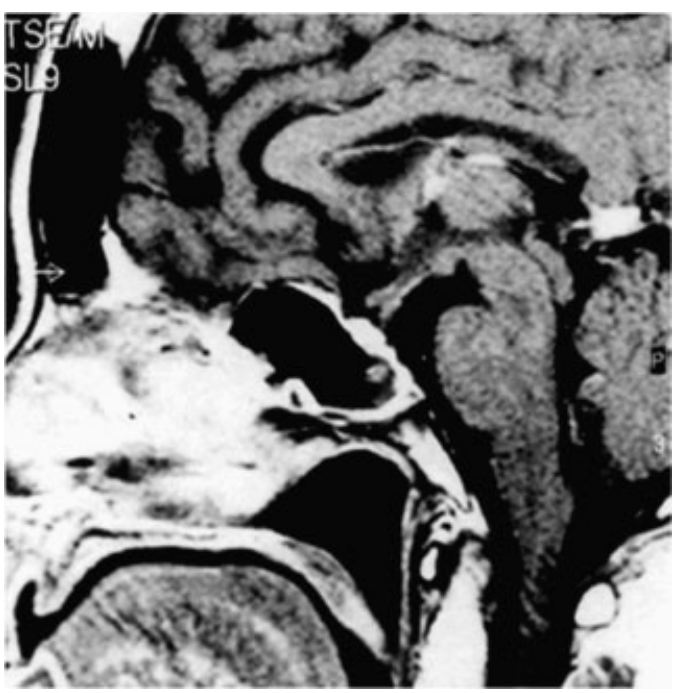

Fig. 12 Postoperative sagittal magnetic resonance image shows the tumor resection.

and decompression of the optic nerve. Radical resection was not done to preserve the other adjacent structures. The patient was referred for further treatment with radiotherapy with a dose of $50 \mathrm{~Gy}$. No recurrence was noted during the postoperative follow-up of 4 years and 2 months (-Fig. 12).

\section{Conclusion}

A substantial prevalence of inflammatory and infectious sphenoid sinus diseases was found, and treatment by endoscopic approach was effective in isolated sphenoid pathologies, whether complicated or not.

Although video endoscopy does not show great sensitivity and specificity for the diagnosis of isolated sphenoid 
pathologies, CT scans and MRI are useful for diagnosis and treatment planning.

\section{References}

1 Grillone GA, Kasznica P. Isolated sphenoid sinus disease. Otolaryngol Clin North Am 2004;37(2):435-451

2 Lew D, Southwick FS, Montgomery WW, Weber AL, Baker AS. Sphenoid sinusitis. A review of 30 cases. N Engl J Med 1983; 309(19):1149-1154

3 Hnatuk LAP, Macdonald RE, Papsin BC. Isolated sphenoid sinusitis: the Toronto Hospital for Sick Children experience and review of the literature. J Otolaryngol 1994;23(1):36-41
4 Metson R, Gliklich RE. Endoscopic treatment of sphenoid sinusitis. Otolaryngol Head Neck Surg 1996;114(6):736-744

5 Martin TJ, Smith TL, Smith MM, Loehrl TA. Evaluation and surgical management of isolated sphenoid sinus disease. Arch Otolaryngol Head Neck Surg 2002;128(12):1413-1419

6 Mra Z, Roach JC, Brook AL. Infectious and neoplastic diseases of the sphenoid sinus-a report of 10 cases. Rhinology 2002;40(1): 34-40

7 Castelnuovo P, Pagella F, Semino L, De Bernardi F, Delù G. Endoscopic treatment of the isolated sphenoid sinus lesions. Eur Arch Otorhinolaryngol 2005;262(2):142-147

8 Socher JA, Cassano M, Filheiro CA, Cassano P, Felippu A. Diagnosis and treatment of isolated sphenoid sinus disease: a review of 109 cases. Acta Otolaryngol 2008;128(9):1004-1010 